To ascertain if patients are aware of the duty to inform the DVLA if they for any reason are not fit to drive.

Background. Risk factors include social, behavior and iatrogenic factors such as social withdrawal, increased likelihood of substance abuse and side effects of anti-psychotic medication.

Method. This trust wide audit involved the random sampling of a total of 71 case notes, 4 case notes per Consultant team in general adult psychiatry and old age psychiatry across Dudley and Walsall sites (total of 3 sites). A data collection tool was developed and included relevant questions regarding fitness to drive. Data were collected between October and December 2019.

Result. 18/49 patients had physical health screening prior to medication initiation.

Conclusion. An important aspect of good medical practice is to educate patients about their condition, this includes their fitness to drive as this can be affected both by their diagnosis and medication. It is clear that clinicians also need to be educated about this responsibility to ensure assessment is performed especially on inpatient discharge.

\section{Improving physical health care for inpatients with eating disorders}

Edward Knights*, Monique Schelhase, Rhys Jones and Lou Burke LYPFT NHSFT

${ }^{*}$ Corresponding author.

doi: 10.1192/bjo.2021.539

Aims. Primary aim - To improve how physical health issues are addressed for inpatients with eating disorders

Secondary aim - To improve efficiency within the MDT

Background. The Yorkshire Centre for Eating Disorders (YCED) is an inpatient unit for the treatment of patients with anorexia and bulimia nervosa. Anorexia nervosa has the highest mortality of all psychiatric disorders with an extensive list of physical manifestations. This project was designed to help better address the physical health concerns of our patients by introducing a primary care style, once weekly clinic that patients could self-refer to.

Method. Questionnaires were designed to assess whether a once weekly physical health clinic would benefit the service.

The clinic was run on a weekly basis from 26th April to 24th June 2019. Follow-up questionnaires were designed and distributed to both patients and staff following this period. Data were analysed with Microsoft Excel to determine if improvement had been made.

Result. $\mathrm{N}=12$ inpatients responded to the initial questionnaires, $\mathrm{n}=2$ were discharged during the 8 week period so were included in the analysis but did not complete the follow-up questionnaire.

$100 \%$ of the staff $(n=8)$ felt a once weekly clinic would benefit their patients. $62 \%(n=5)$ stated they felt distracted from their other duties with physical health requests.

$33 \%(n=4)$ of the inpatient group felt the clinic would benefit them with $67 \%(n=8)$ stating indifference to the idea.

26 appointments were conducted in the physical health clinic with $80 \%(n=8)$ of the service users accessing at least once. $70 \%$ $(n=7)$ stated their physical health concerns had been better addressed since the clinic had been started.

90\% ( $n=9)$ of inpatients and 90\% $(n=9)$ of staff responded that the physical health clinic should remain permanent. $90 \%$ $(n=9)$ of staff stated they had more time for their other duties since the introduction of the clinic.

Prior to the clinic 63\% $(n=5)$ of staff responded that in a typical day they were approached between 2-5 times for physical health requests with the other $37 \%(n=3)$ being approached once.

Following the clinic $80 \%(n=8)$ of staff responded that they were approached once in a typical working day.

Conclusion. The qualitative data from the questionnaires indicated success in both improving patient care and reducing nursing workload.

The physical health clinic has been made a permanent feature on the ward and has been continued by the incoming foundation doctor and ward ANP.

\section{Assessement of a structured technological support intervention on uptake of video consultations}

Tejas Kotwal ${ }^{1 \star}$, Kerushan Thomas ${ }^{1}$, Carlos Escudero King ${ }^{1}$ and Avirup Gupta ${ }^{2}$

${ }^{1}$ king's college london and ${ }^{2}$ South London and Maudsley NHS

Foundation Trust

${ }^{*}$ Corresponding author.

doi: 10.1192/bjo.2021.540

Aims. The coronavirus pandemic has led to an increased reliance on remote patient-clinician interactions, mainly the use of telephone and video consultations. Video consultations are key in psychiatric care, as telephone appointments do not sufficiently allow clinicians to accurately ascertain a patient's mental status and perform a risk assessment. The aim of our quality improvement project was to increase the uptake of video consultations within a community mental health team, focusing on substituting telephone consultations for video.

Method. We accessed Electronic Patient Records to retrospectively quantify the method of contact for 130 consultations delivered over a 4-week period. After collecting baseline data, we conducted focused interviews with 10 care providers, identifying the specific clinician and patient barriers to video uptake that informed our intervention design.

Our intervention consisted of two 4-week Plan, Do, Study, Act (PDSA) cycles.

PDSA 1 involved delivering a focused PowerPoint presentation to the care team, highlighting the benefits of video consultation technology and encouraging clinicians to use it as their primary method of remote communication with patients. Additionally, we conducted qualitative interviews with members of the team to highlight the successes and challenges thus far.

PDSA 2 involved creating a video consultation instructional PDF which highlighted how to operate the technological aspects of both Microsoft Teams and WhatsApp Video Call. This included: how to set-up video calls, accept invitations, and overcome common troubleshooting issues.

The proportion of remote consultations was quantified retrospectively to compare trends in video consultation uptake from baseline to the conclusion of PDSA 2.

Result. Overall, we saw a 15\% increase in video consultations with respect to baseline. The greatest change was attributable to PDSA cycle 1 , which incurred an $8 \%$ increase in video consultation uptake, from $13.85 \%$ to $21.9 \%$. PDSA cycle 2 further increased video consultation uptake by $6.97 \%$, from $21.9 \%$ to $28.87 \%$. Specifically focusing on remote consultations, the proportion conducted with video rather than telephone increased by $17.3 \%$. Interviewed clinicians reported limited financial access, technological fluency, and issues with patient privacy as the most important barriers to the uptake of video consultations.

Conclusion. Our project successfully increased the proportion of consultations conducted by video. This was achieved by targeting 